\title{
The Bound of Entanglement of Superpositions with More Than Two Components
}

\author{
Yang Xiang,* Shi-Jie Xiong, and Fang-Yu Hong \\ National Laboratory of Solid State Microstructures and Department of Physics, \\ Nanjing University, Nanjing 210093, China
}

(Dated: November 9, 2018)

\begin{abstract}
A bipartite quantum state (for two systems in any dimensions) can be decomposed as a superposition of many components. For a superposition of more than two components we prove that there is a bound of the entanglement of the superposition state which can be expressed according to entanglements of its component states. Especially, if the component states are mutually bi-orthogonal, the entanglement of the superposition state can be exactly given in terms of the entanglements of the states being superposed.
\end{abstract}

PACS numbers: 03.67.-a, 03.65.Ta, 03.65.Ud

Superposition and entanglement are two bases of quantum mechanics. For a bipartite pure state, Popescu and Rohrlich [1] proved that there is a unique measure of the entanglement of it, the von Neumann entropy of the reduced state of either of the parties [2]. For example, the entanglement of the bipartite pure state $|\psi\rangle$ is :

$$
E(\psi) \equiv S\left(\operatorname{Tr}_{A}|\psi\rangle\langle\psi|\right)=S\left(\operatorname{Tr}_{B}|\psi\rangle\langle\psi|\right),
$$

where the von Neumann entropy is defined as

$$
S(\rho)=-\operatorname{Tr}(\rho \log \rho) .
$$

In this paper $\log$ denotes $\log _{2}$.

As Linden et al. pointed out [3], entanglement is a global property of a state and it originates from the superposition of different components. For example, four Bell kets are bipartite pure superposition states and each of them is maximally entangled, but every component in the superpositions is unentangled as it can be expressed by a direct product of pure quantum states of the parties. Inversely we can pick out two proper Bell kets to compose a superposition state which is unentangled. In other cases, the forms of two states are almost the same but they do not necessarily have nearly the same entanglement. Thus, there may be some implicit relations between the superposition and the entanglement. We can raise a problem: Given a bipartite superposition state, what is the relation between the entanglement of it and those of the components in the superposition? Linden et al. [3] discussed a state $|\Gamma\rangle$ of two parties and presented a certain decomposition of it as a superposition of two terms $|\Gamma\rangle=\alpha|\psi\rangle+\beta|\phi\rangle$ with $|\alpha|^{2}+|\beta|^{2}=1$. They found an upper bound of the entanglement of $|\Gamma\rangle$ in terms of the entanglements of $|\psi\rangle$ and $|\phi\rangle$. Subsequently, several authors $[5,6,7,8,9,10]$ have discussed some related problems about this issue: in $[5,6,7]$, the authors generalized this result to include different measures of entanglement; in $[8,9]$, the authors discussed the entanglement of superpositions of multipartite states; in [10], the author found tight lower and upper bounds on the entanglement of a superposition of two bipartite states.

In this paper, we generalize the conclusion to a pure bipartite state $|\Psi\rangle$ which is a superposition of more than two $(n>2)$ components

$$
|\Psi\rangle=\alpha_{1}\left|\phi_{1}\right\rangle+\alpha_{2}\left|\phi_{2}\right\rangle+\cdots+\alpha_{n}\left|\phi_{n}\right\rangle
$$

where $\phi_{1}, \phi_{2} \cdots \phi_{n}$ are nonorthogonal and normalized, $\alpha_{1}, \alpha_{2} \cdots \alpha_{n}$ satisfy $\sum_{i=1}^{n} N_{i}^{2}\left|\alpha_{i}\right|^{2}=1$ with $N_{i}$ s being coefficients depending on $n$ as will be discussed below. We present an upper bound on the entanglement of $\Psi$ given by the inequality

$$
\| \alpha_{1}\left|\phi_{1}\right\rangle+\alpha_{2}\left|\phi_{2}\right\rangle+\cdots+\alpha_{n}\left|\phi_{n}\right\rangle \|^{2} \cdot E\left(\alpha_{1} \phi_{1}+\alpha_{2} \phi_{2}+\cdots+\alpha_{n} \phi_{n}\right) \leq \sum_{i=1}^{n} N_{i}^{2}\left|\alpha_{i}\right|^{2} E\left(\phi_{i}\right)+h_{n}
$$

where the notation $E\left(\alpha_{1} \phi_{1}+\alpha_{2} \phi_{2}+\cdots+\alpha_{n} \phi_{n}\right)$ denotes the entanglement of the normalized version of the state $\alpha_{1}\left|\phi_{1}\right\rangle+\alpha_{2}\left|\phi_{2}\right\rangle+\cdots+\alpha_{n}\left|\phi_{n}\right\rangle$, and $h_{n}=$ $-\sum_{i=1}^{n} N_{i}^{2}\left|\alpha_{i}\right|^{2} \log \left(N_{i}^{2}\left|\alpha_{i}\right|^{2}\right)$.
Before embarking on our study, it is worth introducing an inequality first which will be used repeatedly in the following. For a mixed state $\sum_{i=1}^{n} p_{i} \rho_{i}$, the von Neumann 
entropy satisfies [4]

$$
\sum_{i=1}^{n} p_{i} S\left(\rho_{i}\right) \leq S\left(\sum_{i=1}^{n} p_{i} \rho_{i}\right) \leq \sum_{i=1}^{n} p_{i} S\left(\rho_{i}\right)+H
$$

where $H=-\sum_{i=1}^{n} p_{i} \log \rho_{i}$.

In the case that the component states $\phi_{1}, \phi_{2}, \cdots, \phi_{n}$ are mutually biorthogonal we can easily present an exact expression of the entanglement of $\Psi$ in terms of the entanglements of $\phi_{1}, \phi_{2}, \cdots, \phi_{n}[11]$ :

$$
\begin{aligned}
E(\Psi)= & E\left(\alpha_{1} \phi_{1}+\alpha_{2} \phi_{2}+\cdots+\alpha_{n} \phi_{n}\right) \\
= & \left|\alpha_{1}\right|^{2} E\left(\phi_{1}\right)+\left|\alpha_{2}\right|^{2} E\left(\phi_{2}\right)+\cdots \\
& +\left|\alpha_{n}\right|^{2} E\left(\phi_{n}\right)+h_{n}^{\prime}\left(\alpha_{1}, \alpha_{2}, \cdots, \alpha_{n}\right),
\end{aligned}
$$

where

$$
h_{n}^{\prime}\left(\alpha_{1}, \alpha_{2}, \cdots, \alpha_{n}\right)=-\sum_{i=1}^{n}\left|\alpha_{i}\right|^{2} \log \left|\alpha_{i}\right|^{2} .
$$

The definiens of biorthogonal states is that if any two states $\phi_{i}, \phi_{j}(i \neq j)$ in $\phi_{1}, \phi_{2}, \cdots, \phi_{n}$ satisfy

$$
\begin{aligned}
\operatorname{Tr}_{A}\left[\operatorname{Tr}_{B}\left(\left|\phi_{i}\right\rangle\left\langle\phi_{i}\right|\right) \operatorname{Tr}_{B}\left(\left|\phi_{j}\right\rangle\left\langle\phi_{j}\right|\right)\right] & =0 \\
\operatorname{Tr}_{B}\left[\operatorname{Tr}_{A}\left(\left|\phi_{i}\right\rangle\left\langle\phi_{i}\right|\right) \operatorname{Tr}_{A}\left(\left|\phi_{j}\right\rangle\left\langle\phi_{j}\right|\right)\right. & =0
\end{aligned}
$$

we say that $\phi_{1}, \phi_{2} \cdots \phi_{n}$ are mutually biorthogonal, or say that they are biorthogonal states.

Now we discuss the general case that $\phi_{1}, \phi_{2}, \cdots, \phi_{n}$ are nonorthogonal and provide a proof of inequality (4). The case that $\phi_{1}, \phi_{2}, \cdots, \phi_{n}$ are orthogonal (but not biorthogonal) is just a special situation of the general case. At the end we will give another version of (4) for an arbitrary superposition state $|\Psi\rangle=\alpha_{1}\left|\phi_{1}\right\rangle+\alpha_{2}\left|\phi_{2}\right\rangle+\cdots+\alpha_{n}\left|\phi_{n}\right\rangle$, i.e., without the constrained condition $\sum_{i=1}^{n} N_{i}^{2}\left|\alpha_{i}\right|^{2}=1$.

As considered in [3], we think that Alice has a $n$ dimensional Hilbert space $\mathcal{H}_{a}$ besides Hilbert space $\mathcal{H}_{A}$ and introduce an assistant state

$$
\begin{aligned}
|\Lambda\rangle= & \alpha_{1}|1\rangle_{a}\left|\phi_{1}\right\rangle_{A B}+\alpha_{2}|2\rangle_{a}\left|\phi_{2}\right\rangle_{A B} \\
& +\cdots+\alpha_{n}|n\rangle_{a}\left|\phi_{n}\right\rangle_{A B},
\end{aligned}
$$

where the subscripts $a$ denote that $|i\rangle, i=1,2, \cdots, n$ are states in Hilbert space $\mathcal{H}_{a}$ and the subscripts $A B$ denote that $\phi_{1}, \phi_{2} \cdots \phi_{n}$ are bipartite states in Hilbert space $\mathcal{H}_{A} \otimes \mathcal{H}_{B}$. In fact, we can also think that $|\Lambda\rangle$ is a tripartite quantum state of three systems in the Hilbert space $\mathcal{H}_{a} \otimes \mathcal{H}_{A} \otimes \mathcal{H}_{B}$, the two elucidations is equivalent. In the following text we will omit these subscripts. We request that $|i\rangle$ 's $(i=1,2 \cdots, n)$ are mutual orthogonal and normalized, so they become a base of $\mathcal{H}_{a}$. In addition, we require that $\sum_{i=1}^{n}\left|\alpha_{i}\right|^{2}=1$ and $\phi_{1}, \phi_{2}, \cdots, \phi_{n}$ are all normalized, so $|\Lambda\rangle$ is normalized too. Bob's reduced state for $|\Lambda\rangle$ is

$$
\rho_{B}=\sum_{i=1}^{n}\left|\alpha_{i}\right|^{2} \operatorname{Tr}_{A}\left(\left|\phi_{i}\right\rangle\left\langle\phi_{i}\right|\right)
$$

By using inequality (5), we have

$$
\begin{aligned}
S\left(\rho_{B}\right) \leq & \sum_{i=1}^{n}\left|\alpha_{i}\right|^{2} S\left(\operatorname{Tr}_{A}\left(\left|\phi_{i}\right\rangle\left\langle\phi_{i}\right|\right)\right) \\
& +\left(-\sum_{i=1}^{n}\left|\alpha_{i}\right|^{2} \log \left|\alpha_{i}\right|^{2}\right)
\end{aligned}
$$

Now we introduce another normalized and orthogonal base $\left\{\left|\xi_{i}\right\rangle, i=1,2, \cdots, n\right\}$ in Hilbert space $\mathcal{H}_{a}$, and adopt it to express base $\{|i\rangle\}$ as

$$
\begin{aligned}
|1\rangle= & \frac{1}{N_{1}}\left(\left|\xi_{1}\right\rangle+\left|\xi_{2}\right\rangle\right) \\
|2\rangle= & \frac{1}{N_{2}}\left(\left|\xi_{1}\right\rangle-\left|\xi_{2}\right\rangle+\left|\xi_{3}\right\rangle\right) \\
|3\rangle= & \frac{1}{N_{3}}\left(\left|\xi_{1}\right\rangle-\left|\xi_{2}\right\rangle-2\left|\xi_{3}\right\rangle+\left|\xi_{4}\right\rangle\right) \\
& \cdot \\
|i\rangle= & \frac{1}{N_{i}}\left[N_{i-1}|i-1\rangle-\left|\xi_{i}\right\rangle-\left(N_{i-1}^{2}-1\right)\left|\xi_{i}\right\rangle\right. \\
& \left.+\left|\xi_{i+1}\right\rangle\right] \\
& \cdot \\
& \cdot \\
|n-1\rangle= & \frac{1}{N_{n-1}}\left[N_{n-2}|n-2\rangle-\left|\xi_{n-1}\right\rangle\right. \\
& \left.-\left(N_{n-2}^{2}-1\right)\left|\xi_{n-1}\right\rangle+\left|\xi_{n}\right\rangle\right] \\
|n\rangle= & \frac{1}{N_{n}}\left[N_{n-1}|n-1\rangle-\left|\xi_{n}\right\rangle-\left(N_{n-1}^{2}-1\right)\left|\xi_{n}\right\rangle\right] .
\end{aligned}
$$

These $N_{i}$ 's are $|i\rangle$ 's normalization coefficients. They are all positive. It can be easily found that the orthogonality of $|i\rangle$ 's is preserved. For a given $n$, using followed expressions we can easily calculated all $N_{i}$ 's,

$$
\begin{aligned}
N_{1}{ }^{2} & =2 \\
N_{j}^{2} & =\prod_{i=1}^{j-1} N_{i}^{2}+1 \quad 1<j<n \\
N_{n}^{2} & =\prod_{i=1}^{n-1} N_{i}^{2}
\end{aligned}
$$

From Eqs. (9) and (12), we have 


$$
\begin{aligned}
|\Lambda\rangle= & \alpha_{1} \frac{1}{N_{1}}\left(\left|\xi_{1}\right\rangle+\left|\xi_{2}\right\rangle\right)\left|\phi_{1}\right\rangle+\alpha_{2} \frac{1}{N_{2}}\left(\left|\xi_{1}\right\rangle-\left|\xi_{2}\right\rangle+\left|\xi_{3}\right\rangle\right)\left|\phi_{2}\right\rangle+\cdots+\alpha_{i} \frac{1}{N_{i}}\left[\left|\xi_{1}\right\rangle-\cdots-\left(N_{i-1}^{2}-1\right)\left|\xi_{i}\right\rangle+\left|\xi_{i+1}\right\rangle\right]\left|\phi_{i}\right\rangle \\
& +\cdots+\alpha_{n} \frac{1}{N_{n}}\left[\left|\xi_{1}\right\rangle-\cdots-\left(N_{n-1}^{2}-1\right)\left|\xi_{n}\right\rangle\right]\left|\phi_{n}\right\rangle \\
= & \left(\frac{\alpha_{1}}{N_{1}}\left|\phi_{1}\right\rangle+\frac{\alpha_{2}}{N_{2}}\left|\phi_{2}\right\rangle+\cdots+\frac{\alpha_{n}}{N_{n}}\left|\phi_{n}\right\rangle\right)\left|\xi_{1}\right\rangle+\left|C_{2}\right\rangle\left|\xi_{2}\right\rangle+\cdots+\left|C_{n}\right\rangle\left|\xi_{n}\right\rangle
\end{aligned}
$$

where $\left|C_{i}\right\rangle$ 's are some superposition states of $\phi_{1}, \phi_{2}, \cdots, \phi_{n}$. We do not present their explicit expressions here because they will not be requested below. It should be noted that $\left(\frac{\alpha_{1}}{N_{1}}\left|\phi_{1}\right\rangle+\frac{\alpha_{2}}{N_{2}}\left|\phi_{2}\right\rangle+\cdots+\frac{\alpha_{n}}{N_{n}}\left|\phi_{n}\right\rangle\right)$ and $\left|C_{i}\right\rangle$ 's are all not normalized, so we can write $|\Lambda\rangle$ as

$$
\begin{aligned}
|\Lambda\rangle= & \| \frac{\alpha_{1}}{N_{1}}\left|\phi_{1}\right\rangle+\frac{\alpha_{2}}{N_{2}}\left|\phi_{2}\right\rangle+\cdots+\frac{\alpha_{n}}{N_{n}}\left|\phi_{n}\right\rangle \| \cdot\left(\frac{\left(\frac{\alpha_{1}}{N_{1}}\left|\phi_{1}\right\rangle+\frac{\alpha_{2}}{N_{2}}\left|\phi_{2}\right\rangle+\cdots+\frac{\alpha_{n}}{N_{n}}\left|\phi_{n}\right\rangle\right)}{\| \frac{\alpha_{1}}{N_{1}}\left|\phi_{1}\right\rangle+\frac{\alpha_{2}}{N_{2}}\left|\phi_{2}\right\rangle+\cdots+\frac{\alpha_{n}}{N_{n}}\left|\phi_{n}\right\rangle \|}\right)\left|\xi_{1}\right\rangle \\
& +\|\left|C_{2}\right\rangle \| \frac{\left|C_{2}\right\rangle}{\|\left|C_{2}\right\rangle \|}\left|\xi_{2}\right\rangle+\cdots+\|\left|C_{n}\right\rangle \| \frac{\left|C_{n}\right\rangle}{\|\left|C_{n}\right\rangle \|}\left|\xi_{n}\right\rangle .
\end{aligned}
$$

Using the normalization of the $|\Lambda\rangle$ and the orthogonality of the $\left|\xi_{n}\right\rangle$ 's, we obtain

$$
\| \frac{\alpha_{1}}{N_{1}}\left|\phi_{1}\right\rangle+\frac{\alpha_{2}}{N_{2}}\left|\phi_{2}\right\rangle+\cdots+\frac{\alpha_{n}}{N_{n}}\left|\phi_{n}\right\rangle\left\|^{2}+\sum_{i=2}^{n}\right\|\left|C_{i}\right\rangle \|^{2}=1
$$

From Eq. (15) we obtain another expression of Bob's reduced state

$$
\begin{aligned}
\rho_{B}= & \operatorname{Tr}_{A}(|\Lambda\rangle\langle\Lambda|) \\
= & \| \frac{\alpha_{1}}{N_{1}}\left|\phi_{1}\right\rangle+\frac{\alpha_{2}}{N_{2}}\left|\phi_{2}\right\rangle+\cdots+\frac{\alpha_{n}}{N_{n}}\left|\phi_{n}\right\rangle \|^{2} \\
& \times \operatorname{Tr}_{A}\left[\frac{\left(\frac{\alpha_{1}}{N_{1}}\left|\phi_{1}\right\rangle+\frac{\alpha_{2}}{N_{2}}\left|\phi_{2}\right\rangle+\cdots+\frac{\alpha_{n}}{N_{n}}\left|\phi_{n}\right\rangle\right)\left(\frac{\alpha_{1}^{*}}{N_{1}}\left\langle\phi_{1}\right|+\frac{\alpha_{2}^{*}}{N_{2}}\left\langle\phi_{2}\right|+\cdots+\frac{\alpha_{n}^{*}}{N_{n}}\left\langle\phi_{n}\right|\right)}{\| \frac{\alpha_{1}}{N_{1}}\left|\phi_{1}\right\rangle+\frac{\alpha_{2}}{N_{2}}\left|\phi_{2}\right\rangle+\cdots+\frac{\alpha_{n}}{N_{n}}\left|\phi_{n}\right\rangle \|^{2}}\right] \\
& +\|\left|C_{2}\right\rangle\left\|^{2} \operatorname{Tr}_{A}\left(\frac{\left|C_{2}\right\rangle\left\langle C_{2}\right|}{\|\left|C_{2}\right\rangle \|^{2}}\right)+\cdots+\right\|\left|C_{n}\right\rangle \|^{2} \operatorname{Tr}_{A}\left(\frac{\left|C_{n}\right\rangle\left\langle C_{n}\right|}{\|\left|C_{n}\right\rangle \|^{2}}\right) .
\end{aligned}
$$

From Eqs. (5), (11), (17), and (16), we derive

$$
\begin{aligned}
& \| \frac{\alpha_{1}}{N_{1}}\left|\phi_{1}\right\rangle+\frac{\alpha_{2}}{N_{2}}\left|\phi_{2}\right\rangle+\cdots+\frac{\alpha_{n}}{N_{n}}\left|\phi_{n}\right\rangle \|^{2} \\
& \times S\left(\operatorname{Tr}_{A}\left[\frac{\left(\frac{\alpha_{1}}{N_{1}}\left|\phi_{1}\right\rangle+\frac{\alpha_{2}}{N_{2}}\left|\phi_{2}\right\rangle+\cdots+\frac{\alpha_{n}}{N_{n}}\left|\phi_{n}\right\rangle\right)\left(\frac{\alpha_{1}^{*}}{N_{1}}\left\langle\phi_{1}\right|+\frac{\alpha_{2}^{*}}{N_{2}}\left\langle\phi_{2}\right|+\cdots+\frac{\alpha_{n}^{*}}{N_{n}}\left\langle\phi_{n}\right|\right)}{\| \frac{\alpha_{1}}{N_{1}}\left|\phi_{1}\right\rangle+\frac{\alpha_{2}}{N_{2}}\left|\phi_{2}\right\rangle+\cdots+\left.\frac{\alpha_{n}}{N_{n}}\left|\phi_{n}\right\rangle\right|^{2}}\right]\right) \\
& +\|\left|C_{2}\right\rangle\left\|^{2} S\left(\operatorname{Tr}_{A}\left(\frac{\left|C_{2}\right\rangle\left\langle C_{2}\right|}{\|\left|C_{2}\right\rangle \|^{2}}\right)\right)+\cdots+\right\|\left|C_{n}\right\rangle \|^{2} S\left(\operatorname{Tr}_{A}\left(\frac{\left|C_{n}\right\rangle\left\langle C_{n}\right|}{\|\left|C_{n}\right\rangle \|^{2}}\right)\right) \\
\leq & S\left(\rho_{B}\right) \leq \sum_{i=1}^{n}\left|\alpha_{i}\right|^{2} S\left(\operatorname{Tr}_{A}\left(\left|\phi_{i}\right\rangle\left\langle\phi_{i}\right|\right)\right)+\left(-\sum_{i=1}^{n}\left|\alpha_{i}\right|^{2} \log \left|\alpha_{i}\right|^{2}\right) .
\end{aligned}
$$

Since all $\|\left|C_{i}\right\rangle \|^{2} S\left(\operatorname{Tr}_{A}\left(\frac{\left|C_{i}\right\rangle\left\langle C_{i}\right|}{\|\left|C_{i}\right\rangle \|^{2}}\right)\right) \geq 0$, from Eq. (18) we have

$$
\begin{aligned}
& \| \frac{\alpha_{1}}{N_{1}}\left|\phi_{1}\right\rangle+\frac{\alpha_{2}}{N_{2}}\left|\phi_{2}\right\rangle+\cdots+\frac{\alpha_{n}}{N_{n}}\left|\phi_{n}\right\rangle \|^{2} \\
& \times S\left(\operatorname{Tr}_{A}\left[\frac{\left(\frac{\alpha_{1}}{N_{1}}\left|\phi_{1}\right\rangle+\frac{\alpha_{2}}{N_{2}}\left|\phi_{2}\right\rangle+\cdots+\frac{\alpha_{n}}{N_{n}}\left|\phi_{n}\right\rangle\right)\left(\frac{\alpha_{1}^{*}}{N_{1}}\left\langle\phi_{1}\right|+\frac{\alpha_{2}^{*}}{N_{2}}\left\langle\phi_{2}\right|+\cdots+\frac{\alpha_{n}^{*}}{N_{n}}\left\langle\phi_{n}\right|\right)}{\| \frac{\alpha_{1}}{N_{1}}\left|\phi_{1}\right\rangle+\frac{\alpha_{2}}{N_{2}}\left|\phi_{2}\right\rangle+\cdots+\frac{\alpha_{n}}{N_{n}}\left|\phi_{n}\right\rangle \|^{2}}\right]\right)
\end{aligned}
$$




$$
\leq \sum_{i=1}^{n}\left|\alpha_{i}\right|^{2} S\left(\operatorname{Tr}_{A}\left(\left|\phi_{i}\right\rangle\left\langle\phi_{i}\right|\right)\right)+\left(-\sum_{i=1}^{n}\left|\alpha_{i}\right|^{2} \log \left|\alpha_{i}\right|^{2}\right)
$$

Defining $\alpha_{i}^{\prime}=\frac{\alpha_{i}}{N_{i}}$ and noting $\sum_{i=1}^{n}\left|\alpha_{i}\right|^{2}=1$, one has $\sum_{i=1}^{n} N_{i}^{2}\left|\alpha_{i}^{\prime}\right|^{2}=1$. Using $\alpha_{i}^{\prime}$ to express Eq. (19), we finally deduce the inequality (4). When $n=2$, from (13) we learn that $N_{1}^{2}=N_{2}^{2}=2$ and the inequality (4) reduces to the result of [3].

It should be noticed that $E\left(\alpha_{1} \phi_{1}+\alpha_{2} \phi_{2}+\cdots+\alpha_{n} \phi_{n}\right) \neq$ $E\left(\alpha_{1}^{\prime} \phi_{1}+\alpha_{2}^{\prime} \phi_{2}+\cdots+\alpha_{n}^{\prime} \phi_{n}\right)$ except the case of $n=2$, the reason is that usually $\left(\left|\alpha_{i}^{\prime}\right|^{2} /\left|\alpha_{j}^{\prime}\right|^{2}\right)=\left(\left|\alpha_{i}\right|^{2} /\left|\alpha_{j}\right|^{2}\right) \times$ $\left(N_{j}^{2} / N_{i}^{2}\right) \neq\left(\left|\alpha_{i}\right|^{2} /\left|\alpha_{j}\right|^{2}\right)$.

In the case that $\phi_{1}, \phi_{2}, \cdots, \phi_{n}$ are orthogonal but not biorthogonal, for deducing the inequality (4) we only need to replace $\| \alpha_{1}\left|\phi_{1}\right\rangle+\alpha_{2}\left|\phi_{2}\right\rangle+\cdots+\alpha_{n}\left|\phi_{n}\right\rangle \|^{2}$ with $\left(\left|\alpha_{1}\right|^{2}+\left|\alpha_{2}\right|^{2}+\cdots+\left|\alpha_{n}\right|^{2}\right)$.

In the expression (3) and the inequality (4), the constrained condition $\sum_{i=1}^{n} N_{i}^{2}\left|\alpha_{i}\right|^{2}=1$ obviously makes the function $h_{n}$ positive, and does not add any restrictions on the superposition itself. For example, for any superposition state $|\Psi\rangle=\alpha_{1}\left|\phi_{1}\right\rangle+\alpha_{2}\left|\phi_{2}\right\rangle+\cdots+\alpha_{n}\left|\phi_{n}\right\rangle$, multiplying it by a constant $\frac{1}{\sqrt{\sum_{i=1}^{n} N_{i}^{2}\left|\alpha_{i}\right|^{2}}}$, we have a new state $|\Psi\rangle^{\prime}=\alpha_{1}^{\prime}\left|\phi_{1}\right\rangle+\alpha_{2}^{\prime}\left|\phi_{2}\right\rangle+\cdots+\alpha_{n}^{\prime}\left|\phi_{n}\right\rangle$. These $\alpha_{i}^{\prime}$ 's satisfy $\sum_{i=1}^{n} N_{i}^{2}\left|\alpha_{i}^{\prime}\right|^{2}=1$. Recalling that $E(\Psi)$ in inequality (4) denotes the entanglement of the normalized version of state $\Psi, E(\Psi)=E\left(\Psi^{\prime}\right)$ and we can use inequality (4) to discuss arbitrary superposition states. It is easy to find that for arbitrary $|\Psi\rangle=\alpha_{1}\left|\phi_{1}\right\rangle+\alpha_{2}\left|\phi_{2}\right\rangle+\cdots+\alpha_{n}\left|\phi_{n}\right\rangle$ without the constrained condition $\sum_{i=1}^{n} N_{i}^{2}\left|\alpha_{i}\right|^{2}=1$, we have an inequality

$$
\| \alpha_{1}\left|\phi_{1}\right\rangle+\alpha_{2}\left|\phi_{2}\right\rangle+\cdots+\alpha_{n}\left|\phi_{n}\right\rangle \|^{2} \cdot E\left(\alpha_{1} \phi_{1}+\alpha_{2} \phi_{2}+\cdots+\alpha_{n} \phi_{n}\right) \leq \sum_{i=1}^{n} N_{i}^{2}\left|\alpha_{i}\right|^{2} E\left(\phi_{i}\right)+h_{n}^{\prime}
$$

where $h_{n}^{\prime}=-\sum_{i=1}^{n} N_{i}^{2}\left|\alpha_{i}\right|^{2} \log \left(N_{i}^{2}\left|\alpha_{i}\right|^{2}\right)+\log \left(\sum_{i=1}^{n} N_{i}^{2}\left|\alpha_{i}\right|^{2}\right) \cdot \sum_{i=1}^{n} N_{i}^{2}\left|\alpha_{i}\right|^{2}$.

In fact, if we multiply $|\Psi\rangle$ by a different constant $\frac{1}{\sqrt{\sum_{i=1}^{n} N_{i}^{\prime 2}\left|\alpha_{i}\right|^{2}}}$, where $\left\{N_{i}^{\prime}\right\}$ is a different order of $\left\{N_{i}\right\}$, we have the same state $|\Psi\rangle^{\prime}$ and a different constrained condition $\sum_{i=1}^{n} N_{i}^{\prime}{ }^{2}\left|\alpha_{i}^{\prime}\right|^{2}=1$, as a consequence we will obtain a different inequality (20). Considering this effect, for any superposition state, the inequality (20) should be moderated as

$$
\| \alpha_{1}\left|\phi_{1}\right\rangle+\alpha_{2}\left|\phi_{2}\right\rangle+\cdots+\alpha_{n}\left|\phi_{n}\right\rangle \|^{2} \cdot E\left(\alpha_{1} \phi_{1}+\alpha_{2} \phi_{2}+\cdots+\alpha_{n} \phi_{n}\right) \leq \min _{\left\{N_{i}\right\}}\left\{\sum_{i=1}^{n} N_{i}^{2}\left|\alpha_{i}\right|^{2} E\left(\phi_{i}\right)+h_{n}^{\prime}\right\}
$$

where $\min _{\left\{N_{i}\right\}}$ means that the lowest bound is taken over all possible sets of $\left\{N_{i}\right\}$, associated with different values of $\alpha_{i}$ and $\phi_{i}$ in the sum and $h_{n}^{\prime}$ on the right-hand side of Eq. (21).

In summary, we present an upper bound on the entanglement of superposition states with more than two components. The bound expressed in inequality (4) with arbitrary $\left\{N_{i}\right\}$ most likely not be the best one. For many cases, we find that the bound is loose. We suppose that there may be two reasons for the looseness of the bound: (i) In the derivation of inequality (4) we have used the relation $\sum_{i=1}^{n} p_{i} S\left(\rho_{i}\right) \leq \sum_{i=1}^{n} p_{i} S\left(\rho_{i}\right)+H$ and so the difference between conditions for two equalities Eq. (5) should make the bound hard to be achieved. (ii) In the derivation of Eq. (19), we have dropped the terms such as $\|\left|C_{i}\right\rangle \|^{2} S\left(\operatorname{Tr}_{A}\left(\frac{\left|C_{i}\right\rangle\left\langle C_{i}\right|}{\|\left|C_{i}\right\rangle \|^{2}}\right)\right)$, and this may reduce the value of the left-hand side in inequality (4). In [10], the author has shown that the bound in [3] is not tight and given a tighter upper bound for the case of superposition with two components. In the case of $n=2$, our inequality (4) reduces to the result of [3], so the result of Gour [10] implies that there may be a better inequal- 
ity for the entanglement of superposition with more than two components. This may be a task for future work.

Acknowledgments We would like to thank the referee who reminded us to note the effect which result in Eq. (21). This work was supported by National Foundation of Natural Science in China Grant Nos. 60676056 and 10474033, and by the China State Key Projects of Basic Research (2005CB623605 and 2006CB0L1000).

* Electronic address: njuxy@sina.com

[1] S. Popescu, D. Rohrlich, Phys. Rev. A 56(1997) R3319.

[2] C.H. Bennett, H.J. Bernstein, S. Popescu, B. Schumacher, Phys. Rev. A 53(1996) 2.
[3] N.Linden, S. Popescu and J.A. Smolin, Phys.Rev.Lett. 97(2006) 100502.

[4] See for example, M.A. Nielsen and I.L. Chuang, "Quantum Computation and Quantum Information", CUP, Cambridge (2000).

[5] Chang-shui Yu, X. X. Yi, and He-shan Song, Phys. Rev. A 75(2007) 022332.

[6] Yong-Cheng Ou and Heng Fan, Phys. Rev. A 76, 022320(2007).

[7] J. Niset and N. J. Cerf, Phys. Rev. A 76, 042328(2007).

[8] D. Cavalcanti, M. O. Terra Cunha and A. Acín, Phys. Rev. A 76, 042329(2007).

[9] Wei Song, Nai-Le Liu and Zeng-Bing Chen, Phys. Rev. A 76, 054303(2007).

[10] Gilad Gour, Phys. Rev. A 76, 052320(2007).

[11] An explicit calculation can be seen in arXiv: quantph/0701188 